

\section{Program at a Glance}

\section{Fri. March 26 8:50-16:00 \\ Annual Meeting of the Japan Endocrine Society \\ 17:00-18:35 \\ Opening Ceremony (Main Hall) \\ $18: 45-$ \\ Welcome Reception (Sakura \& Swan) \\ We have the honor to have the attendance of Their Majesties the Emperor and Empress at the Opening Ceremony. All participants are requested to enter the main hall by 16:30 and to present their Photo ID at the Security Check. \\ Walking in/out during the Opening Ceremony (16:30-18:35) is strictly prohibited.}

\begin{tabular}{|c|c|c|c|c|c|c|c|c|c|c|c|c|c|c|c|}
\hline & \multicolumn{9}{|c|}{ Sat. March 27} & \multicolumn{6}{|c|}{ Sun. March 28} \\
\hline $\begin{array}{l}\text { Room } \\
\text { No. }\end{array}$ & $\begin{array}{l}8: 25- \\
9: 10\end{array}$ & $\begin{array}{l}9: 20- \\
11: 00\end{array}$ & $\begin{array}{l}11: 10- \\
12: 00\end{array}$ & $\begin{array}{l}\text { 12:10- } \\
13: 00\end{array}$ & \begin{tabular}{|c|}
$13: 10-$ \\
$14: 20$
\end{tabular} & $\begin{array}{l}14: 20- \\
16: 00\end{array}$ & $\begin{array}{l}16: 10- \\
16: 55\end{array}$ & $\begin{array}{l}16: 55- \\
17: 40\end{array}$ & $\begin{array}{l}17: 50- \\
19: 20\end{array}$ & $\begin{array}{l}8: 25- \\
9: 10\end{array}$ & $\begin{array}{l}9: 20- \\
11: 00\end{array}$ & $\begin{array}{l}11: 10- \\
12: 00\end{array}$ & $\begin{array}{l}\text { 12:10- } \\
13: 00\end{array}$ & $\begin{array}{l}3: 10- \\
14: 20\end{array}$ & $\begin{array}{l}14: 20- \\
16: 00\end{array}$ \\
\hline $\begin{array}{l}\text { Room } 1 \\
\text { Main Hall }\end{array}$ & $\begin{array}{c}\text { PL } \\
1\end{array}$ & $\begin{array}{l}\text { SY } \\
15-2\end{array}$ & \multicolumn{2}{|c|}{$\begin{array}{l}\text { Panel Discussion } \\
\text { 11:10-12:40 }\end{array}$} & & $\begin{array}{l}\text { SY } \\
3-2\end{array}$ & $\begin{array}{c}\mathrm{PL} \\
\mathbf{2}\end{array}$ & $\begin{array}{l}\text { PL } \\
3\end{array}$ & & $\begin{array}{l}\mathrm{PL} \\
4\end{array}$ & $\begin{array}{l}\text { SY } \\
2-6\end{array}$ & $\begin{array}{l}\text { JESMeister } \\
\text { Award } \\
\text { lecture }\end{array}$ & & & $\begin{array}{l}\text { SY } \\
3-1\end{array}$ \\
\hline $\begin{array}{l}\text { Room } 2 \\
\text { Annex } 1\end{array}$ & & $\begin{array}{l}\text { SY } \\
1-1\end{array}$ & $\underset{1}{\text { MTP }}$ & & & $\begin{array}{l}\text { SY } \\
9-6\end{array}$ & & & & & $\begin{array}{c}\text { SY } \\
14-3\end{array}$ & $\begin{array}{c}\text { MTP } \\
11\end{array}$ & & \multicolumn{2}{|c|}{$\begin{array}{l}\text { Public Forum } \\
\text { 14:00-16:30 }\end{array}$} \\
\hline $\begin{array}{l}\text { Room3 } \\
\text { Annex 2 }\end{array}$ & & $\begin{array}{l}\text { SY } \\
11-2\end{array}$ & $\underset{2}{\text { MTP }}$ & $\begin{array}{c}\text { LS } \\
1\end{array}$ & & $\begin{array}{l}\text { SY } \\
4-4\end{array}$ & & & $\begin{array}{c}\text { SS } \\
1\end{array}$ & & $\begin{array}{l}\text { SY } \\
8-1\end{array}$ & $\begin{array}{c}\text { MTP } \\
12\end{array}$ & $\begin{array}{l}\text { LS } \\
6\end{array}$ & & $\begin{array}{l}\text { SY } \\
9-2\end{array}$ \\
\hline$\underset{\mathrm{A}}{\mathrm{Room} 4}$ & & $\begin{array}{l}\text { SY } \\
5-5\end{array}$ & $\underset{3}{\text { MTP }}$ & $\begin{array}{c}\text { LS } \\
2\end{array}$ & & $\begin{array}{c}\text { SY } \\
15-3\end{array}$ & & & & & $\begin{array}{l}\text { SY } \\
4-2\end{array}$ & $\begin{array}{c}\text { MTP } \\
13\end{array}$ & $\begin{array}{l}\text { LS } \\
7\end{array}$ & & $\begin{array}{l}\text { SY } \\
7-1\end{array}$ \\
\hline$\stackrel{\text { Room5 }}{\mathrm{D}}$ & & $\begin{array}{l}\text { SY } \\
1-3\end{array}$ & $\underset{4}{\mathrm{MTP}}$ & $\begin{array}{l}\text { LS } \\
3\end{array}$ & & $\begin{array}{l}\text { SY } \\
1-2\end{array}$ & & & & & $\begin{array}{l}\text { SY } \\
7-2\end{array}$ & $\underset{14}{\text { MTP }}$ & $\begin{array}{c}\text { LS } \\
8\end{array}$ & & $\begin{array}{l}\text { SY } \\
8-6\end{array}$ \\
\hline$\underset{E}{R o o m 6}$ & & $\begin{array}{l}\text { SY } \\
3-4\end{array}$ & $\underset{5}{\text { MTP }}$ & $\begin{array}{l}\text { LS } \\
4\end{array}$ & & $\begin{array}{l}\text { SY } \\
5-1\end{array}$ & & & & & $\begin{array}{l}\text { SY } \\
15-1\end{array}$ & $\begin{array}{c}\text { MTP } \\
15\end{array}$ & $\begin{array}{c}\text { LS } \\
9\end{array}$ & & $\begin{array}{l}\text { SY } \\
9-3\end{array}$ \\
\hline $\begin{array}{c}\text { Room } 7 \\
\text { B-1 }\end{array}$ & & $\begin{array}{l}\text { SY } \\
4-6\end{array}$ & $\underset{6}{\text { MTP }}$ & $\begin{array}{l}\text { LS } \\
5\end{array}$ & & $\begin{array}{l}\text { SY } \\
1-6\end{array}$ & & & & & $\begin{array}{l}\text { SY } \\
1-5\end{array}$ & $\begin{array}{l}\text { MTP } \\
16\end{array}$ & $\begin{array}{l}\text { LS } \\
10\end{array}$ & & $\begin{array}{l}\text { SY } \\
4-1\end{array}$ \\
\hline $\begin{array}{c}\text { Room } 8 \\
\text { B-2 }\end{array}$ & & $\begin{array}{l}\text { SY } \\
7-4\end{array}$ & $\underset{7}{\text { MTP }}$ & & & $\begin{array}{l}\text { SY } \\
1-4\end{array}$ & & & & & $\begin{array}{l}\text { SY } \\
5-3\end{array}$ & $\begin{array}{c}\text { MTP } \\
17\end{array}$ & $\begin{array}{l}\text { LS } \\
11\end{array}$ & & $\begin{array}{l}\text { SY } \\
2-1\end{array}$ \\
\hline$\underset{C-1}{\operatorname{Room} 9}$ & & $\begin{array}{l}\text { SY } \\
12-2\end{array}$ & $\underset{8}{\text { MTP }}$ & $\begin{array}{c}\mathrm{OH} \\
1\end{array}$ & & $\begin{array}{c}\text { SY } \\
10-1\end{array}$ & & & & & $\begin{array}{c}\text { SY } \\
10-3\end{array}$ & $\begin{array}{c}\text { MTP } \\
18\end{array}$ & $\underset{5}{\mathrm{OH}}$ & & $\begin{array}{l}\text { SY } \\
5-2\end{array}$ \\
\hline$\underset{C-2}{\operatorname{Room} 10}$ & & $\begin{array}{l}\text { SY } \\
1-7\end{array}$ & $\underset{9}{\text { MTP }}$ & $\underset{2}{\mathrm{OH}}$ & & $\begin{array}{c}\text { SY } \\
10-4\end{array}$ & & & & & $\begin{array}{l}\text { SY } \\
1-9\end{array}$ & $\begin{array}{c}\text { MTP } \\
19\end{array}$ & $\begin{array}{c}\mathrm{OH} \\
6\end{array}$ & & $\begin{array}{c}\text { SY } \\
10-5\end{array}$ \\
\hline$\underset{\mid}{\operatorname{Room} 11}$ & & $\begin{array}{l}\text { SY } \\
3-7\end{array}$ & $\begin{array}{l}0 \\
1\end{array}$ & $\underset{3}{\mathrm{OH}}$ & & $\begin{array}{l}\text { SY } \\
3-5\end{array}$ & & & & & $\begin{array}{c}\text { SY } \\
11-6\end{array}$ & $\begin{array}{l}0 \\
9\end{array}$ & $\underset{7}{\mathrm{OH}}$ & & $\begin{array}{l}\text { SY } \\
3-6\end{array}$ \\
\hline$\underset{J}{R o o m} 12$ & & $\begin{array}{c}\text { SY } \\
14-2\end{array}$ & $\begin{array}{l}0 \\
2\end{array}$ & $\underset{4}{\mathrm{OH}}$ & & $\begin{array}{l}\text { SY } \\
7-8\end{array}$ & & & & & $\begin{array}{c}\text { SY } \\
8-11\end{array}$ & $\begin{array}{c}0 \\
10\end{array}$ & $\underset{8}{\mathrm{OH}}$ & & $\begin{array}{c}\text { SY } \\
15-5\end{array}$ \\
\hline$\underset{K}{\operatorname{Room} 13}$ & & $\begin{array}{c}\text { SY } \\
13-5\end{array}$ & $\begin{array}{l}0 \\
3\end{array}$ & & & $\begin{array}{c}\text { SY } \\
15-6\end{array}$ & & & & & $\begin{array}{c}\text { SY } \\
15-4\end{array}$ & $\begin{array}{l}0 \\
11\end{array}$ & & & $\begin{array}{c}\text { SY } \\
14-1\end{array}$ \\
\hline $\begin{array}{c}\text { Room } 14 \\
554\end{array}$ & & $\begin{array}{l}\text { SY } \\
4-3\end{array}$ & $\begin{array}{l}0 \\
4\end{array}$ & & & $\begin{array}{l}\text { SY } \\
1-8\end{array}$ & & & & & $\begin{array}{l}\text { SY } \\
4-7\end{array}$ & $\begin{array}{c}\text { MTP } \\
20\end{array}$ & & & $\begin{array}{l}\text { SY } \\
11-7\end{array}$ \\
\hline$\underset{555}{\operatorname{Room} 15}$ & & $\begin{array}{l}\text { SY } \\
7-5\end{array}$ & $\begin{array}{c}\text { MTP } \\
10\end{array}$ & & & $\begin{array}{l}\text { SY } \\
11-1\end{array}$ & & & & & $\begin{array}{c}\text { SY } \\
12-3\end{array}$ & $\underset{21}{\text { MTP }}$ & & & $\begin{array}{c}\text { SY } \\
12-4\end{array}$ \\
\hline $\begin{array}{c}\text { Room } 16 \\
510\end{array}$ & & JES 1 & $\begin{array}{l}0 \\
5\end{array}$ & & & JES 2 & & & & & JES 4 & $\begin{array}{c}0 \\
12\end{array}$ & & & JES 6 \\
\hline $\begin{array}{c}\text { Room } 17 \\
501\end{array}$ & & $\begin{array}{c}0 \\
6 / 7\end{array}$ & $\begin{array}{l}0 \\
8\end{array}$ & & & JES 3 & & & & & JES 5 & $\begin{array}{l}0 \\
13\end{array}$ & & & JES 7 \\
\hline Event Hall & $\begin{array}{c}\text { Poster } \\
\text { Mounting } \\
8: 00- \\
9: 00\end{array}$ & & $\begin{array}{l}\text { Poster } \\
\text { Display }\end{array}$ & & $\begin{array}{c}\text { Poster } \\
\text { Discussion } \\
\text { 13:10-10 } \\
\text { 14:20 }\end{array}$ & $\begin{array}{l}\text { Poster } \\
\text { Display }\end{array}$ & $\begin{array}{c}\text { Poster } \\
\text { Removal } \\
16: 00- \\
17: 00\end{array}$ & & & $\begin{array}{c}\text { Poster } \\
\text { Mounting } \\
8: 00- \\
9: 00\end{array}$ & & $\begin{array}{l}\text { Poster } \\
\text { Display }\end{array}$ & & $\begin{array}{c}\text { Poster } \\
\text { Disussion } \\
13: 10- \\
14: 20\end{array}$ & $\begin{array}{l}\text { Poster } \\
\text { Display }\end{array}$ \\
\hline Event Hall & & & & & Exhibition & & & & & & & Exhil & ition & & \\
\hline
\end{tabular}


PL: Plenary Lectures

MTP: Meet-the-Professor

SY: Symposia

JES: JES-Sponsored Symposia

$\mathrm{OH}$ : Oral Hot Topics

O: Oral Sessions

P: Poster Presentations

LS: Luncheon Seminars

SS: Satellite Symposia

SY1: Diabetes Mellitus

SY2: Obesity and Adiposcience

SY3: Peptides

SY4: Thyroid

SY5: Bone and Mineral Metabolism

SY6: Endocrine Surgery

SY7: Pituitary/Neuroendocrinology

SY8: Steroid Receptors

SY9: Cardiovascular Endocrinology and Metabolism

SY10: Tumor
SY11: Reproduction and

SY12: Regeneration

SY13: Pediatric Endocrinology

SY14: Signal Transduction

SY15: Adrenal

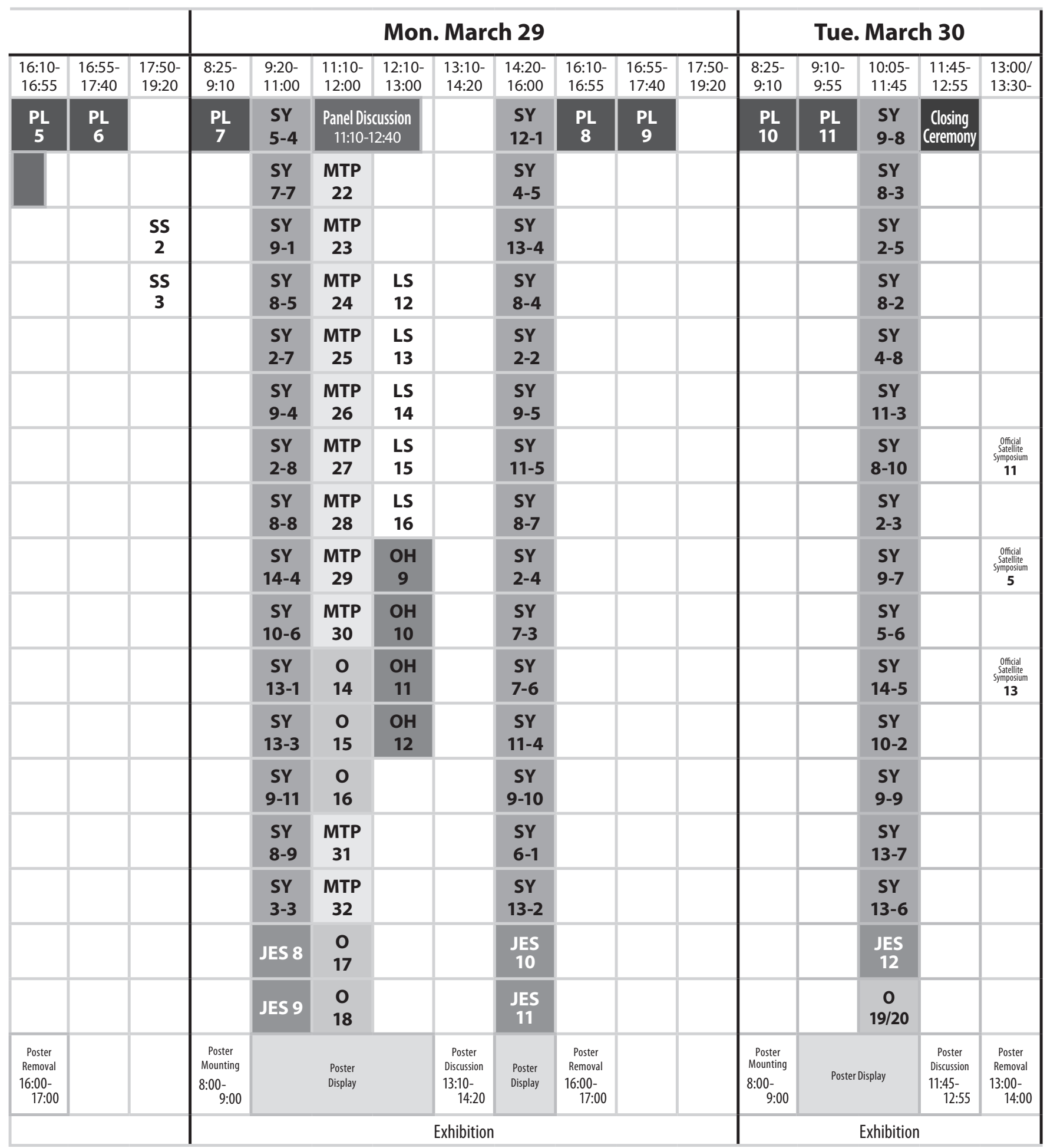

